\title{
Urban Distribution Optimization of Electric Vehicles Considering the Distribution of Charging Piles and Carbon Emission
}

\author{
Hui Hu${ }^{1, *}$, Yong Guo ${ }^{2}$, Xiongfei He ${ }^{1}$, Hai Lin ${ }^{1}$, Biyao Wang ${ }^{1}$ and Nuonuo Xue ${ }^{1,3}$ \\ ${ }^{I}$ School of automobile, Chang'an University, Xi'an, Shaanxi, China \\ ${ }^{2} X i$ 'an Railway Station, Xi'an, Shaanxi, China \\ ${ }^{3}$ School of computing, Teesside University,Middlesbrough, United Kingdom
}

Received 1 February 2019; Accepted 14 April 2019

\begin{abstract}
The increasing demand of urban distribution aggravates urban congestion and environmental pollution. In view of the growing serious environmental problems, the urban distribution of electric vehicles (EVs) can alleviate the pollution caused by vehicle emissions to a certain extent. However, in practical application, this urban distribution is affected by the scarce distribution of public charging piles and the limited mileage per charge, which bring difficulties to the operation scheduling. An optimization method considering the distribution of public charging piles and carbon emissions was proposed to improve the efficiency of the urban distribution of EVs. With the minimum total distribution cost as the optimization objective, an optimization model based on the distribution of the public charging pile network and considering the restriction of mileage per charge was established. Hill-climbing algorithm was introduced in accordance with the requirements and characteristics of the optimization model to improve the standard genetic algorithm (SGA) and its local solving capability. The feasibility and validity of the model and hill-climbing genetic algorithm (HCGA) were verified by solving small- and large-scale numerical examples. Results demonstrate that the optimization model and HCGA proposed can effectively provide optimized solutions for the urban distribution of EVs. The designed HCGA has a better searching capability than SGA. For the small-scale numerical example, the average total cost of the feasible solutions obtained by HCGA is $18.2 \%$ less than that by SGA, whereas for the large-scale example, the cost is $6 \%$ less than that by SGA. This study provides references for saving on the distribution cost and guiding the urban distribution scheduling and optimization of EVs.
\end{abstract}

Keywords: Distribution; Vehicle routing problem (VRP); Standard genetic algorithm (SGA)

\section{Introduction}

With the rapid development of China's economy and urbanization, e-commerce has become increasingly prosperous, thereby increasing urban distribution. In China, the government calls for green logistics, which requires increasing the proportion of green vehicles in the urban distribution system. Developing green low-carbon logistics has a positive effect on alleviating urban environmental problems. The January 2019 sales report of BYD, a leading manufacturer of electric vehicles (EVs) in China, compared with the statistics of the same period last year, indicates that the sales of EVs have increased by $38516.67 \%$, whereas the sales of fuel vehicles have decreased by $64.95 \%$. Under the influence of policy support, environmental awareness enhancement, and energy shortage, the popularization and application of EVs are becoming a growing trend. However, numerous problems, such as the limited mileage per charge caused by battery capacity, insufficient distribution of public charging piles, and long charging time of EVs, hamper the practical application of EVs. Therefore, considering the distribution of public charging piles and mileage per charge, this study focuses on the urban distribution optimization of

\footnotetext{
*E-mail address: huhvi0414@outlook.com

ISSN: $1791-2377$ @ 2019 Eastern Macedonia and Thrace Institute of Technology. All rights reserved. doi:10.25103/jestr.122.19
}

EVs in the low-carbon logistics environment, which can guide third-party logistics enterprises in managing and operating the urban distribution of EVs.

Recently, several studies have been conducted on the urban distribution optimization of EVs [1,2]. However, numerous problems remain, such as the oversimplification of constraints of the optimization model and low solving quality of algorithms, which lead to the unreasonable scheduling scheme of EVs. Therefore, combining the technical characteristics and development reality of EVs, constructing a scientific and practical optimization model for the urban distribution of EVs, and designing an efficient algorithm are urgent problems to be solved.

For this reason, an optimization model for the urban distribution of EVs is established, considering the distribution of public charging piles, mileage per charge, and carbon emissions of EVs. An improved genetic algorithm (GA) is designed for the optimized solution of the model, which can provide reference for the distribution operation and management of EVs of third-party logistics enterprises.

\section{State of the art}

Dantzig and Ramser [3] first proposed the vehicle routing problem (VRP) in 1959, which attracted considerable 
attention worldwide. Many scholars continue to analyze the problem for practical applications. Golden et al. [4] studied a VRP with uncertain driving cost and multiple vehicle types. Considering the diversified customer demands, complexity of the distribution network, and other uncertainties, VRP with time window has emerged. Grosso [5] constructed a VRP model with access time windows from the actual situation of prohibiting trucks from entering the central city in Europe and discussed the effect of time constraints on transportation costs. Salma Naccache et al. [6] investigated a simultaneous delivery problem with time windows and developed a hybrid adaptive large neighborhood search with improved operation through branch and bound and heuristic algorithms. Xie [7] analyzed a VRP with multiple soft time windows. On the basis of the number of vehicles, driving cost, and penalty cost of the deviation from the time window, an optimization model was established considering the constraints of vehicle loading capacity and maximum route length. $\mathrm{Wu}$ [8] examined a VRP with multi-resource constraints of vehicle battery and loading capacity, established static and dynamic models under the constraints, and used CPLEX12.6.2 to find the solution. VRP is a typical NP-hard problem. To improve the efficiency of the algorithm searching capability, $\mathrm{Xu}$ [9] evaluated a dynamic VRP and designed a standard ant colony algorithm (ACA) to fuse the improved $\mathrm{k}$-means and crossover to obtain improved solutions. However, existing studies mostly regard minimum cost and time or maximum profit as the optimization objectives and consider the constraints of vehicle loading capacity, time window, and demand satisfaction. The algorithms designed are mainly traditional and simple.

Recently, many advanced technologies have been used to enhance the efficiency and safety of vehicles [10]. With the popularity of the low-carbon concept, energy conservation and environmental protection are considered in VRP modeling, which is gradually becoming a research trend. Zhang et al. [11] incorporated the fuel, carbon emission, and vehicle use costs into modeling and designed an improved tabu search algorithm (RS-TS) to find the solution. The battery capacity of EVs will limit the mileage per charge. However, existing studies have not considered this problem. Liu [12] optimized the network of urban charging piles for EVs, which is important for EV distribution research. Keskin et al. [13] investigated the VRP of EVs with time window, regarded EV charge as 0-1 mixed integer linear programming, and developed an adaptive large neighborhood search algorithm. Yao et al. [14] proposed a multi-objective collaborative planning strategy to solve the optimization problem of integrated power distribution and charging systems for EVs. When energy consumption and emissions of the vehicles are introduced into the VRP model, the GVRP model is proposed [15-18]. Goel [19] established an optimization model with the objectives of minimum route length and delivery time, considering carbon emission and energy utilization. However, the mileage per charge restricted by battery capacity was neglected. The most widely used algorithms for solving VRP are GA [20] and ACA [21]. The drawbacks of ACA include low convergence rate and susceptibility to local optimum solutions. In addition, the search is prone to stagnation. Lang et al. [22] combined the GA and hill-climbing algorithm to obtain an improved optimal solution, which provided reference for the algorithm design in the present study. Hence, an optimization model for the urban distribution of EVs considering carbon cost and EV characteristics is developed, and an improved GA is designed to solve the optimization model.

In summary, existing studies mainly focus on improving the objective function and constraints of the VRP model, such as expanding a single objective to multiple objectives and considering soft or hard time windows in the constraints. Thus, such studies neglect the mileage per charge and charging time of EVs, which causes the solutions to be inconsistent with reality. Therefore, a VRP optimization model considering the distribution of public charging piles and the restriction of driving mileage is constructed, and an improved GA is designed to solve the model.

The remainder of this study is organized as follows. Section 3 establishes the VRP optimization model of EVs. Section 4 designs the hill-climbing genetic algorithm (HCGA) and proves its effectiveness by testing small- and large-scale numerical examples. Finally, Section 5 summarizes the conclusions and presents future research directions.

\section{Methodology}

The charging electricity of EVs is transferred to the environmental cost of carbon emissions and included in the total distribution cost of the objective function. In this study, a VRP optimization model of EVs based on a public charging pile network, which considers the constraints of vehicle loading capacity, mileage per charge, and carbon emission cost, is established.

\subsection{Model establishment}

A GVRP model considering loading capacity and mileage per charge is established on the basis of the technical characteristics of EVs. This study assumes that each vehicle starts from the distribution center with full power. During distribution, if the vehicle power cannot meet the distribution requirements, then it will look for a public charging pile to charge. If the customer's demand is higher than the vehicle loading capacity, then the vehicle will return to the distribution center to load for the subsequent distribution. The maximum driving mileage for EVs is set to avoid the second charge. If the distribution distance exceeds the maximum driving mileage of the vehicle, then it will find a nearby charging pile to charge and then continue to work. When calculating the total distribution cost, the average fixed cost of single delivery, the variable cost of single delivery, the cost of electricity consumption, and the environmental cost caused by the carbon emissions of upstream enterprises are considered.

\subsubsection{Model assumptions}

1) The EVs only deliver goods and do not pick-up goods during delivery.

2) Only one distribution center exists, which is also the origin and destination of all EVs.

3) All EVs have the same type and indicators.

4) For EVs that complete the delivery, when the power of the vehicles cannot meet the requirements, they will charge at nearby charging piles and are fully charged every time.

5) The customers are deterministic, that is, a static VRP is analyzed in this study.

6) Vehicle charging is accomplished at public charging piles or in the distribution center. The charging mode is fast

charging, that is, ignoring the cost of charging time.
7) Transporting goods between customers is not permitted. 


\subsubsection{Model formulation}

To meet the demands of $n$, the distribution task is assigned by the distribution center $S_{0} . Q_{p}$ is the loading capacity of $\mathrm{EVs} ; q_{j}$ represents the demand of goods by customer $j$. The demand by the distribution center is 0 . Under the premise of satisfying all customers' demands, the model sets the minimum total cost as the optimization objective. For $S_{0}$, the minimum vehicle number needed $k$ is expressed as

$$
k=\operatorname{int}\left|\frac{\sum_{j \in N} q_{j}}{\partial Q_{p}}\right|+1
$$

where $\partial$ indicates the full loading rate of vehicles. Therefore, the GVRP model of EVs is as follows:

$$
\begin{aligned}
\operatorname{MinZ}_{1}= & A_{1} k+A_{2} \sum_{k=1}^{m} \sum_{j=1}^{n} \sum_{i=0}^{n} X_{i j k} \cdot d_{i j}+A_{3} \sum_{k=1}^{m} \sum_{j=1}^{n} \sum_{i=0}^{n} X_{i j k} \cdot d_{i j} \mathrm{l} \\
& +\gamma B_{4} \lambda \sum_{k=1}^{m} \sum_{j=1}^{n} \sum_{i=0}^{n} X_{i j k} \cdot d_{i j} \mathrm{l}
\end{aligned}
$$

$$
\text { s.t. } \sum_{k=1}^{m} \sum_{j=1}^{n+p} X_{i j k}=1, \forall i \in C \text {, }
$$

$$
\sum_{i \in C_{k}^{l}} q_{j} \sum_{j \in N_{k}^{i}} X_{i j k} \leq Q_{P}, \forall k \in V
$$

$\sum_{j \in N_{k}^{\prime}} X_{0 j k}=1, \sum_{i \in N_{k}^{\prime}} X_{i 0 k}=1, \forall k \in V, \forall l \in L_{k}$,

$$
\sum_{i \in O_{k}^{*}} \sum_{j \in O_{k}^{*}} X_{i j k} \cdot d_{i j} \leq p_{0}, \forall k \in V, \forall r \in R_{K},
$$

$\sum_{i \in N_{k}^{l}} \sum_{j \in N_{k}^{t}} X_{i j k} \cdot d_{i j} \leq p_{\max }, \forall k \in V, \forall l \in L_{k}$.

\section{$X_{i j k}$ is the decision variable.}

Equation (2) presents the objective function with the minimum total cost. In Equation (3), the distribution center serves each customer only once. In Equation (4), the goods loaded cannot exceed the maximum loading capacity of the vehicles. In Equation (5), the departure and return depots of each vehicle are distribution centers. In Equation (6), the driving distance of a vehicle should not exceed the mileage per charge. In Equation (7), the driving distance should not exceed the maximum driving mileage.

The following describes the meaning of symbols:

$V$ : set of EVs, $V=\{k\}, k=1,2,3 \cdots m . m$ is the number of EVs in the distribution center and a variable to be determined;

$C:$ set of customers, $C=\{i\}, i=1,2,3 \cdots n$, where $n$ denotes the number of customers;

$N$ : set of vertices, including $n$ customer points to be served and $p$ public charging pile points;

$O_{k}^{r}$ : vertex set of the $r^{\text {th }}$ open sub-path of vehicle $k$ (open sub-path indicates that vehicle $k$ moves from one charging pile to the other);
$N_{k}^{l}$ : vertex set of the $l^{\text {th }}$ closed sub-path of vehicle $k$ (closed sub-path indicates that vehicle $k$ has departed from distribution center $S_{0}$ and returned to $S_{0}$ );

$C_{k}^{l}$ : customer vertex set of the $l^{\text {th }}$ closed sub-path of vehicle $k$;

$q_{j}:$ demand for goods of customer $j$;

$Q_{P}$ : loading capacity of EVs;

$d_{i j}$ : distance from $i$ to $j$;

$P_{0}$ : mileage per charge;

$P_{\max }:$ maximum driving mileage;

$A_{1}$ : average fixed cost per delivery of EVs;

$A_{2}$ : variable cost per km of EVs;

$A_{3}$ : unit cost per KWh;

$\mathrm{l}$ : power consumption per $\mathrm{km}$ of EVs $\left(k w \cdot h \cdot \mathrm{km}^{-1}\right)$;

$\lambda$ : carbon emission coefficient of electric energy $\left(k g(k w \cdot h)^{-1}\right)$

$\gamma:$ percent of thermal power for unit electricity amount.

\subsection{Algorithm design}

A VRP, including a single distribution center and multicustomer depots, is studied. When designing the algorithm, several factors, such as loading capacity, mileage per charge, and distribution of public charging piles, must be considered. Therefore, an algorithm with comprehensive search capability and high search tolerance should be designed when solving the model. Considering these factors, GA is adopted to solve the model. GA is widely used because of its powerful global searching capability and simple process. However, the standard genetic algorithm (SGA) has the disadvantage of poor local search capability. Hence, the hillclimbing algorithm is introduced to improve the local search capability of SGA. The hill-climbing algorithm, also known as a local search method, is a single direction search algorithm based on domain search technology, which can improve the quality of the solution. This algorithm has a strong local search capability, but its global search capability is insufficient. Hence, the combination of hill-climbing algorithm and SGA, namely, HCGA, can learn from one another and effectively overcome the weakness of SGA in local search capability. Figure 1 presents the flow chart of HCGA.

The adopted particle representation scheme is direct and easy to realize; besides, it no longer needs to prescribe the number of communities of a network, because it can automatically determine this.

\subsubsection{Chromosome design and population initialization} When designing the GA, $C=\{i\},(i=1,2,3 \cdots n)$ is randomly generated by using the demand depots as the genes and adopting integer coding. Each permutation represents a chromosome of length $n$. For example, a randomly generated chromosome $\{1,2,5,4,3\}$ represents a chromosome, where the gene sequence is the distribution order of a vehicle. Considering the vehicle loading capacity, each customer is added to the distribution route one by one, until the total demand of goods attains the vehicle loading capacity. When the vehicle cannot meet the next customer's demand, the distribution route will be reconstructed from this customer depot. This process is repeated until all 
Hui Hu, Yong Guo, Xiongfei He, Hai Lin, Biyao Wang and Nuonuo Xue/

Journal of Engineering Science and Technology Review 12 (2) (2019) 135 - 142

customers are assigned to the EVs and all routes are identified.

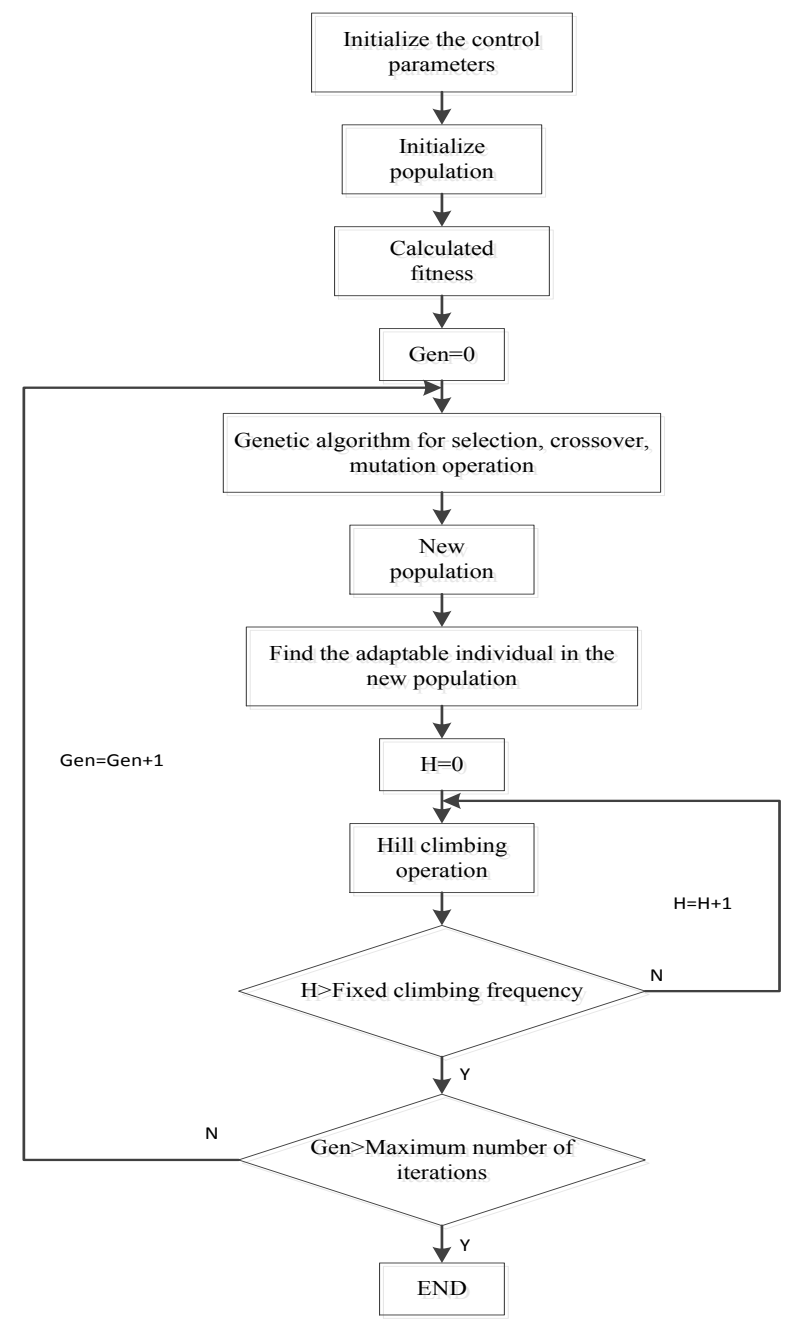

Fig. 1. Flow chart of HCGA

\subsubsection{Fitness function}

Fitness function is determined in accordance with the objective function and is the only criterion for natural selection. The fitness function is non-negative. The objective of the optimization model is minimum cost; therefore, the reciprocal of the objective function is used as the fitness function.

\subsubsection{Genetic operator}

\section{1) Selection}

Selection is the operation of the survival of the fittest among individuals in a population. The fitness determines the probability of chromosomes being inherited by the next generation. The higher the chromosome fitness, the more likely it is to be inherited, and vice versa. The selection method adopted in this study is roulette selection.

The detailed steps are as follows: first, the fitness value of all individuals in population $F_{i}$ is calculated. Then, the probability that chromosome $i$ is selected and passed on to the next population $p_{i}=F_{i} / \sum F_{i}$ is calculated. Finally, on the basis of a certain random rule, the chromosome individuals are selected to enter the next generation.

\section{2) Crossover}

Crossover refers to the exchange of genes between two pairs of chromosomes in a way that creates two new individuals. The new individual retains the basic characteristics of the father generation. Two-point crossover is adopted, which is generally implemented in two steps. The first step is to pair individuals randomly in a group. The second step is to set two crossover nodes randomly in the paired individuals, then exchange the genes between the crossover nodes, and modify the two obtained chromosomes. 3) Mutation

The GA can improve the local search capability through mutation, maintain the population diversity, and prevent the premature phenomenon. In this study, the chromosome is constructed in the form of a customer code, and the charging effect is considered in decoding. Therefore, when mutating, changing the customer distribution order is considered to improve the group diversity. The mutational mode of mutual reciprocity is adopted, that is, two points in the chromosome are randomly selected and their values are exchanged.

\section{4) Hill-climbing operation}

In comparison with the SGA, the hill-climbing operation is used after mutation, and a fixed number of hill climbing is introduced. The detailed process is as follows. In the optimal individuals of each generation, we select a gene segment, reverse the sequence of the gene segment, repeatedly perform the operation, obtain the fitness value each time, and record the maximum fitness and its corresponding individuals. If the fitness value is higher than that before the climbing operation, new individuals are accepted; otherwise, the operation is invalid.

\subsubsection{Decoding method}

The algorithm decoding process mainly includes two parts: 1) Divide code $S$ in accordance with the constraints of loading capacity and mileage per charge, and the detailed method is as follows:

(1) $i=1$;

(2) Start route, and 0 is the distribution center;

(3) Attempt to add the first point in code $S$ to $R_{i}$. If the loading capacity and mileage of the vehicle are satisfied, then proceed to the step (4); otherwise, $i=i+1$ and repeat step (2);

(4) Delete the first code of $S$. If $S$ is empty, then proceed to step (5); otherwise, repeat step (3);

(5) Output each sub-path.

2) Cut each obtained route to meet the mileage constraint. For each route,

(1) $k=1, d_{1}=0$;

(2) Calculate the current driving distance $d_{1}=d_{1}+d_{2}$, where $d_{2}$ is the distance between nodes $k$ and $k+1$.

Then, calculate $\quad d_{3}=d_{1}+d_{4}$ where $d_{4}=\min ($ dmat6) . dmat 6 represents the distance between node $k+1$ and all charging piles. If $d_{4} \leq L_{\max }$, where $L_{\max }$ is the maximum length permitted, then proceed to step (3); otherwise, proceed to (4);

(3) For $k=k+1$, if $k>n$, then proceed to step (5);

(4) For $d_{1}=0$, add the nearest charging pile into the path, and repeat step (3);

(5) Output the result. 
Hui Hu, Yong Guo, Xiongfei He, Hai Lin, Biyao Wang and Nuonuo Xue/

Journal of Engineering Science and Technology Review 12 (2) (2019) 135 - 142

3.2.5 Parameter setting and algorithm termination conditions

The HCGA parameters mainly include the climbing times, population size, termination algebra, and probability of different genetic operations. The population size, number of iterations, and times of hill climbing depend on the actual situation. The range of crossover probability is generally between 0.4 and 0.99 , and the range of mutation probability is generally between 0.0001 and 0.1 . The condition for the termination of the algorithm is that when the iteration number reaches the maximum, the algorithm is terminated and the optimal route is obtained.

\section{Result Analysis and Discussion}

\subsection{Case introduction}

Company A is responsible for urban distribution in a certain area of the city. The goods are delivered to the distribution center by the upstream distributor and then delivered to each supermarket. The company plans to use the EVs for urban distribution, and the vehicle costs 250,000 RMB. Considering the subsidies for the EVs, the company can acquire 50,000 RMB from the Chinese government and 25,000 RMB from the local government. If the subsidies are considered, on the basis of the service period of EVs, the fixed transport cost $A_{1}=42 R M B /$ vehicle $\cdot$ time, and the variable cost per vehicle $A_{2}=0.60 R M B / \mathrm{km}$. The average driving speed is $50 \mathrm{~km} / \mathrm{h}$, and the electricity consumption $\mathrm{l}=0.5 \mathrm{kw} \cdot \mathrm{h} / \mathrm{km}$. The electricity price $A_{3}=0.82 \mathrm{RMB} / \mathrm{kw} \cdot \mathrm{h}$. The electric energy conversion coefficient $\lambda=0.94$. The proportion of thermal power generation $\gamma=0.72$. The environmental cost caused by carbon emission $B_{4}=0.315 R M B / \mathrm{kg}$. The loading capacity of the EVs is 1.6 t. The mileage per charge $P_{0}=80 \mathrm{~km}$, and the maximum driving mileage $P_{\max }=180 \mathrm{~km}$. Then, small- and large-scale numerical examples are used to solve the model.

\subsection{Small-scale numerical example}

The coordinate of the distribution center is $(30,30)$. The customer coordinates can be obtained on the basis of the actual position of each customer, as shown in Table 1.

Fast charging is adopted to minimize charging time. Table 2 shows the location of the fast charging piles within the distribution area. The experiments on the two kinds of benchmark networks have demonstrated the effectiveness of the proposed PSO-net algorithm for network clustering. In the next step, we test the algorithms on some real-world networks.

Table 1. Customer coordinates and demand

\begin{tabular}{l|l|l|l|l|l|l|l|l|l|l}
\hline Custome-rdepot & $\mathbf{1}$ & $\mathbf{2}$ & $\mathbf{3}$ & $\mathbf{4}$ & $\mathbf{5}$ & $\mathbf{6}$ & $\mathbf{7}$ & $\mathbf{8}$ & $\mathbf{9}$ & $\mathbf{1 0}$ \\
\hline $\mathbf{x}$ & 7.5 & 12 & 15 & 25.5 & 3 & 55.5 & 57 & 30 & 30 & 21 \\
$\mathbf{y}$ & 36 & 4.5 & 15 & 22.5 & 49.5 & 1.5 & 3 & 10.5 & 39 & 45 \\
\hline Demand & 0.4 & 0.2 & 0.2 & 0.4 & 0.4 & 0.2 & 0.4 & 0.2 & 0.2 & 0.2 \\
\hline Custome-rdepot & $\mathbf{1 1}$ & $\mathbf{1 2}$ & $\mathbf{1 3}$ & $\mathbf{1 4}$ & $\mathbf{1 5}$ & $\mathbf{1 6}$ & $\mathbf{1 7}$ & $\mathbf{1 8}$ & $\mathbf{1 9}$ & $\mathbf{2 0}$ \\
\hline $\mathbf{x}$ & 55.5 & 22.5 & 7.5 & 48 & 24 & 15 & 25.5 & 6 & 9 & 30 \\
$\mathbf{y}$ & 39 & 28.5 & 33 & 18 & 45 & 12 & 42 & 12 & 22.5 & 15 \\
\hline Demand & 0.2 & 0.4 & 0.4 & 0.4 & 0.2 & 0.4 & 0.4 & 0.2 & 0.4 & 0.2 \\
\hline
\end{tabular}

Table 2. Charging pile coordinates

\begin{tabular}{l|l|l|l|l|l}
\hline Charging piles & C1 & C2 & C3 & C4 & C5 \\
\hline $\mathrm{X}$ & 36 & 18 & 16.5 & 42 & 25.5 \\
\hline $\mathrm{Y}$ & 48 & 52.5 & 7.5 & 21 & 37.5 \\
\hline
\end{tabular}

MATLAB (R2016a) is used to find the solution for the optimization model. In the designed algorithm, the population size is set to 100 , the maximum evolutionary algebra to 500 , the crossover probability to 0.7 , the mutation probability to 0.09 , and the climbing times to 20 . The solution is randomly determined 10 times, and Table 3 presents the results (in two decimal places).

Table 3. Results of small-scale numerical examples by the HCGA

\begin{tabular}{l|l|l|l|l|l|l|l|l|l|l}
\hline & $\mathbf{1}$ & $\mathbf{2}$ & $\mathbf{3}$ & $\mathbf{4}$ & $\mathbf{5}$ & $\mathbf{6}$ & $\mathbf{7}$ & $\mathbf{8}$ & $\mathbf{9}$ & $\mathbf{1 0}$ \\
\hline $\begin{array}{l}\text { Cost (RMB) } \\
\begin{array}{l}\text { Iterations of the } \\
\text { optimized solution } \\
\text { (times) }\end{array}\end{array}$ & 581.39 & 590.25 & 567.58 & 566.32 & 580.63 & 616.03 & 553.82 & 570.38 & 576.42 & 593.10 \\
Running time (s) & 56.12 & 454 & 458 & 317 & 477 & 184 & 450 & 307 & 299 & 419 \\
\hline
\end{tabular}

Table 3 shows that the feasible solutions are obtained within these 10 solutions by the HCGA. The average total cost is $579.60 \mathrm{RMB}$, and the average running time is $55.93 \mathrm{~s}$ The seventh solution has the highest quality with a total cost of 553.82 RMB. Table 4 shows the detailed distribution scheme.

To compare the quality of solutions, SGA is used to solve the model randomly for 10 times, with a maximum number of 500 iterations, a population size of 100 , a crossover probability of 0.7 , and a mutation probability of 0.05 . Table 5 presents the results.

The SGA also obtains feasible solutions, with an average total cost of $709.35 \mathrm{RMB}$, which is $18.2 \%$ more than that by the HCGA. The running time is $12.36 \mathrm{~s}$. The seventh solution has the highest quality, and the total distribution cost is $673.59 \mathrm{RMB}$.

Figure 2 shows the optimization process for solving the small-scale example by using the SGA and HCGA 
Hui Hu, Yong Guo, Xiongfei He, Hai Lin, Biyao Wang and Nuonuo Xue/

Journal of Engineering Science and Technology Review 12 (2) (2019) 135 - 142

Table 4. Optimization scheme for the urban distribution of EVs (small scale)

\begin{tabular}{l|c|c}
\hline Route & Distance (km) & \\
\hline $0 \rightarrow 3 \rightarrow 19 \rightarrow 16 \rightarrow 18 \rightarrow 2 \rightarrow \mathrm{C} 3 \rightarrow 20 \rightarrow 0$ & 97.3677 & Cost (RMB) \\
$0 \rightarrow 8 \rightarrow 6 \rightarrow 7 \rightarrow 14 \rightarrow \mathrm{C} 4 \rightarrow 11 \rightarrow 0$ & 122.4056 & 150.7204 \\
$0 \rightarrow 4 \rightarrow 12 \rightarrow 15 \rightarrow 17 \rightarrow 9 \rightarrow 0$ & 49.7851 & 178.6777 \\
$0 \rightarrow 13 \rightarrow 1 \rightarrow 5 \rightarrow 10 \rightarrow 0$ & 75.9762 & 97.5898 \\
\hline
\end{tabular}

Table 5. Results of small-scale examples solved by the SGA

\begin{tabular}{|c|c|c|c|c|c|c|c|c|c|c|}
\hline No. & 1 & 2 & 3 & 4 & 5 & 6 & 7 & 8 & 9 & 10 \\
\hline Cost (RMB) & 681.86 & 717.52 & 710.44 & 724.36 & 707.80 & 673.59 & 726.15 & 691.96 & 737.82 & 721.90 \\
\hline $\begin{array}{l}\text { Iterations of the } \\
\text { optimal solution } \\
\text { (times) }\end{array}$ & 331 & 299 & 348 & 90 & 450 & 453 & 324 & 393 & 48 & 311 \\
\hline Running time (s) & 12.67 & 12.61 & 11.98 & 12.45 & 12.08 & 11.86 & 12.29 & 12.94 & 12.17 & 12.53 \\
\hline
\end{tabular}

As shown as Figure 2, in the entire evolution process, except for a few search stages, the quality of solutions of the SGA is lower than that of the HCGA, and its convergence speed is not as fast as the latter. Under the same search times, the optimization and convergence of the SGA are less efficient than that of the HCGA.

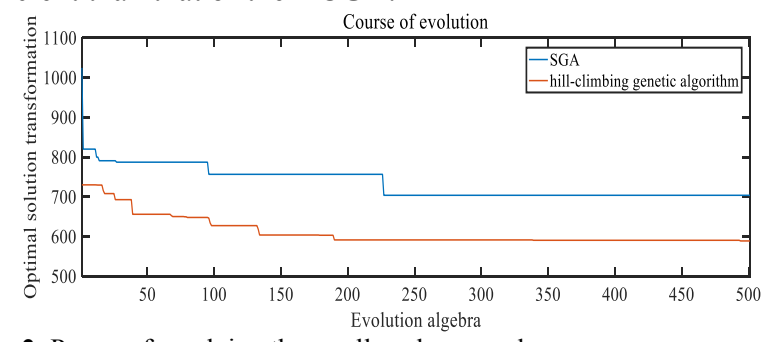

Fig. 2. Process for solving the small-scale example

\subsection{Large-scale numerical example}

To verify the generality of the algorithm further, 30 customers are added into the small-scale example. EVs are assumed to charge only once during the distribution. Therefore, considering the increase in distance between customers, the other type of EVs is used with a rated loading capacity of $2.0 \mathrm{t}$. The mileage per charge is $120 \mathrm{~km}$, and the maximum driving mileage is $220 \mathrm{~km}$. Table 6 presents the customer information.

Table 6. New customer coordinates and demand

\begin{tabular}{l|l|l|l|l|l|l|l|l|l|l}
\hline Customer depot & $\mathbf{2 1}$ & $\mathbf{2 2}$ & $\mathbf{2 3}$ & $\mathbf{2 4}$ & $\mathbf{2 5}$ & $\mathbf{2 6}$ & $\mathbf{2 7}$ & $\mathbf{2 8}$ & $\mathbf{2 9}$ & $\mathbf{3 0}$ \\
\hline $\mathbf{x}$ & 15 & 12 & 15 & 42 & 25.5 & 55.5 & 51 & 37.5 & 42 & 28.5 \\
$\mathbf{y}$ & 36 & 22.5 & 30 & 22.5 & 54 & 42 & 31.5 & 16.5 & 24 & 52.5 \\
Demand & 0.4 & 0.2 & 0.2 & 0.4 & 0.4 & 0.2 & 0.2 & 0.4 & 0.2 & 0.2 \\
\hline
\end{tabular}

Table 6. New customer coordinates and demand (continued)

\begin{tabular}{l|l|l|l|l|l|l|l|l|l|l}
\hline Customer depot & $\mathbf{3 1}$ & $\mathbf{3 2}$ & $\mathbf{3 3}$ & $\mathbf{3 4}$ & $\mathbf{3 5}$ & $\mathbf{3 6}$ & $\mathbf{3 7}$ & $\mathbf{3 8}$ & $\mathbf{3 9}$ & $\mathbf{4 0}$ \\
\hline $\mathbf{x}$ & 25.5 & 24 & 22.5 & 43.5 & 27 & 13.5 & 52.5 & 34.5 & 22.5 & 12 \\
$\mathbf{y}$ & 34.5 & 43.5 & 48 & 9 & 34.5 & 12 & 16.5 & 15 & 55.5 & 13.5 \\
Demand & 0.2 & 0.4 & 0.4 & 0.2 & 0.4 & 0.4 & 0.2 & 0.4 & 0.4 & 0.2 \\
\hline
\end{tabular}

Table 6. New customer coordinates and demand (continued)

\begin{tabular}{l|l|l|l|l|l|l|l|l|l|l}
\hline Customer depot & $\mathbf{4 1}$ & $\mathbf{4 2}$ & $\mathbf{4 3}$ & $\mathbf{4 4}$ & $\mathbf{4 5}$ & $\mathbf{4 6}$ & $\mathbf{4 7}$ & $\mathbf{4 8}$ & $\mathbf{4 9}$ & $\mathbf{5 0}$ \\
\hline $\mathbf{x}$ & 42 & 51 & 34.5 & 3 & 45 & 9 & 19.5 & 37.5 & 43.5 & 34.5 \\
$\mathbf{y}$ & 39 & 6 & 12 & 25.5 & 30 & 43.5 & 28.5 & 37.5 & 4.5 & 21 \\
Demand & 0.4 & 0.2 & 0.2 & 0.4 & 0.4 & 0.2 & 0.2 & 0.4 & 0.2 & 0.2 \\
\hline
\end{tabular}

Table 7. Charging pile coordinates

\begin{tabular}{l|l|l|l|l|l|l|l|l|l}
\hline Charging piles & $\mathbf{C 1}$ & $\mathbf{C 2}$ & $\mathbf{C 3}$ & $\mathbf{C 4}$ & $\mathbf{C 5}$ & $\mathbf{C 6}$ & $\mathbf{C 7}$ & $\mathbf{C 8}$ & $\mathbf{C 9}$ \\
$\mathbf{x}$ & 36 & 18 & 16.5 & 42 & 25.5 & 51 & 40.5 & 22.5 & 45 \\
$\mathbf{y}$ & 48 & 52.5 & 7.5 & 21 & 37.5 & 37.5 & 19.5 & 42 & 15 \\
\hline
\end{tabular}

Similarly, we design HCGA in MATLAB (R2016a) to solve the model. The population size is set to 100 , the maximum evolutionary algebra is 500, the crossover probability is 0.7 , the mutation probability is 0.09 , and the climbing times are 20. A total of 10 solutions are randomly performed, and Table 8 presents the results (in one decimal place).

Table 8. Results of large-scale examples by the HCGA

\begin{tabular}{l|l|l|l|l|l|l|l|l|l|l}
\hline No. & $\mathbf{1}$ & $\mathbf{2}$ & $\mathbf{3}$ & $\mathbf{4}$ & $\mathbf{5}$ & $\mathbf{6}$ & $\mathbf{7}$ & $\mathbf{8}$ & $\mathbf{9}$ & $\mathbf{1 0}$ \\
\hline Cost (RMB) & 1675.6 & 1668.3 & 1693.2 & 1673.1 & 1659.2 & 1664.7 & 1697.5 & 1604.1 & 1701.9 & 1670.3 \\
\hline $\begin{array}{l}\text { Iterations of the } \\
\text { optimal solution } \\
\text { (times) }\end{array}$ & 362 & 179 & 249 & 494 & 391 & 168 & 390 & 420 & 242 & 405 \\
\hline Running time (s) & 113.0 & 112.1 & 113.5 & 111.8 & 111.5 & 112.7 & 113.6 & 113.8 & 112.4 & 113.1 \\
\hline
\end{tabular}


Hui Hu, Yong Guo, Xiongfei He, Hai Lin, Biyao Wang and Nuonuo Xue/

Journal of Engineering Science and Technology Review 12 (2) (2019) 135 - 142

Table 8 shows that feasible solutions are obtained within the 10 solutions by the HCGA. The average total cost is $1670.8 \mathrm{RMB}$, and the average running time is $112.75 \mathrm{~s}$. The eighth solution has the highest quality, and the total distribution cost is 1604.1 RMB. Table 9 displays the detailed scheme.

Table 9. Optimization scheme for the urban distribution of EVs (large scale)

\begin{tabular}{l|c|c}
\hline Route & Distance (km) & Cost (RMB) \\
\hline $0 \rightarrow 27 \rightarrow 2 \rightarrow 22 \rightarrow 15 \rightarrow 33 \rightarrow \mathrm{C} 5 \rightarrow 29 \rightarrow 45 \rightarrow 43 \rightarrow 0$ & 193.6818 & 258.2643 \\
$0 \rightarrow 12 \rightarrow 9 \rightarrow 3 \rightarrow 16 \rightarrow 36 \rightarrow 18 \rightarrow 34 \rightarrow \mathrm{C} 8 \rightarrow 0$ & 151.7400 & 211.4322 \\
$0 \rightarrow 7 \rightarrow 48 \rightarrow 11 \rightarrow 26 \rightarrow \mathrm{C} 6 \rightarrow 24 \rightarrow 31 \rightarrow 40 \rightarrow 0$ & 192.5179 & 256.9647 \\
$0 \rightarrow 35 \rightarrow 20 \rightarrow 4 \rightarrow 38 \rightarrow 28 \rightarrow 30 \rightarrow 0$ & 108.6115 & 163.2752 \\
$0 \rightarrow 8 \rightarrow 50 \rightarrow 10 \rightarrow 39 \rightarrow 41 \rightarrow \mathrm{C} 7 \rightarrow 42 \rightarrow 6 \rightarrow 0$ & 175.8775 & 238.3841 \\
$0 \rightarrow 1 \rightarrow 19 \rightarrow 49 \rightarrow 14 \rightarrow 37 \rightarrow \mathrm{C} 4 \rightarrow 47 \rightarrow 0$ & 71.05043 & 198.8859 \\
$0 \rightarrow 17 \rightarrow 32 \rightarrow 13 \rightarrow 44 \rightarrow 23 \rightarrow 0$ & 101.6861 & 121.3424 \\
$0 \rightarrow 21 \rightarrow 5 \rightarrow 25 \rightarrow 46 \rightarrow 0$ & & 155.5422 \\
\hline
\end{tabular}

To compare the quality of solutions, the SGA is used to solve the model randomly for 10 times, with a maximum number of 500 iterations, a total group size of 100 , a crossover probability of 0.7 , and a mutation probability of 0.05 . Table 10 presents the results.

Table 10. Results of large-scale examples solved by SGA

\begin{tabular}{l|l|l|l|l|l|l|l|l|l|l}
\hline Times & $\mathbf{1}$ & $\mathbf{2}$ & $\mathbf{3}$ & $\mathbf{4}$ & $\mathbf{5}$ & $\mathbf{6}$ & $\mathbf{7}$ & $\mathbf{8}$ & $\mathbf{9}$ & $\mathbf{1 0}$ \\
\hline Cost (RMB) & 1749.1 & 1771.5 & 1754.7 & 1781.1 & 1775.2 & 1801.7 & 1746.4 & 1751.1 & 1792.5 & 1810.7 \\
\hline $\begin{array}{l}\text { Iterations of the } \\
\text { optimal solution } \\
\text { (times) }\end{array}$ & 354 & 298 & 143 & 93 & 272 & 242 & 425 & 361 & 186 & 293 \\
\hline Running time (s) & 39.7 & 39.5 & 40.1 & 39.8 & 39.5 & 39.8 & 39.7 & 39.3 & 39.5 & 40.0 \\
\hline
\end{tabular}

As shown in Table 10, the SGA also obtains feasible solutions with an average value of $1773.4 \mathrm{RMB}$, which is $6 \%$ more than that by the HCGA. The running time is approximately $39 \mathrm{~s}$, which is approximately $1 / 3$ of the time of the HCGA. The seventh solution has the highest quality, and its total distribution cost is 1746.4 RMB.

Similarly, Figure 3 exhibits the optimization process for solving the large-scale example by using the SGA and HCGA.

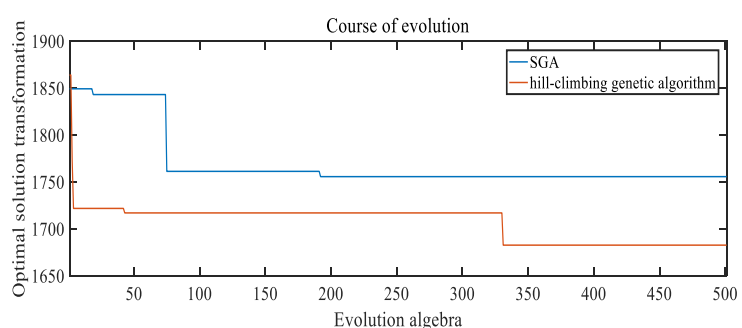

Fig. 3. Process for solving the large-scale example

The optimization and convergence capability of the SGA are inferior to those of the HCGA. With the iterations, the solution quality of the two algorithms improves continuously, but the corresponding running time also increases. Through the comparative analysis, the following characteristics of the HCGA are summarized as follows:

1) Both algorithms can obtain feasible solutions, and the quality of solutions increases with the number of iterations. However, in view of the quality of feasible solutions and the optimized solution, the HCGA is better than the SGA, which reflects that the HCGA has better optimization performance.

2) In the entire iteration process, except for a few stages, the solution quality of the HCGA is better than that of the SGA. Only a few iterations are required to obtain highquality solutions, which reflects that the HCGA has better convergence capability than the SGA.

3) As the process of the HCGA is more complex than that of the SGA, additional running time is required for the
HCGA under the same computer condition (CPU, i5-5200u; memory, 4 GB). However, with the improvement of computer performance, the speed of HCGA will also improve.

\section{Conclusions}

An optimization method is investigated to improve the economical efficiency of the urban distribution of EVs and ensure the practicability of the scheduling scheme. When establishing the model, carbon cost is added to the optimization objective, and realistic factors, such as the distribution of public charging piles and the mileage per charge of EVs, are considered. Then, an improved GA is designed to solve the model. The conclusions are as follows:

1) The energy consumption of EVs is converted to carbon cost and considered in the objective function. When designing the constraints, the loading capacity, mileage per charge, and distribution of public charging piles are considered simultaneously, so that the model will reflect the actual distribution scenario and to ensure that the distribution optimization scheme is close to reality.

2) By solving small- and large-scale numerical examples, the quality of optimized solution obtained by the HCGA is found to be higher than that by the SGA, which proves that the HCGA has a better performance. Given the complexity of the HCGA, its computing time is long, but with the improvement of computer performance, its computing time is within acceptable range. An efficient and reliable algorithm guarantees the accuracy of the optimized solution and helps achieve an efficient scheduling scheme.

This study considers the practical constraints of the mileage per charge of EVs and insufficient distribution of public charging piles, which have a certain reference value for the practical operation of logistics enterprises. However, in practical logistics distribution, many types of VRPs exist. 
Hui Hu, Yong Guo, Xiongfei He, Hai Lin, Biyao Wang and Nuonuo Xue/

Journal of Engineering Science and Technology Review 12 (2) (2019) 135 - 142

In the future, we will further examine the complex distribution routing problems of EVs, such as time window constraints, simultaneous pick-up and delivery, and transfer of goods between customers. This study focuses on the static VRP, which can be extended to dynamic VRP in the future.

\section{Acknowledgements}

This work was supported by the National Natural Science Foundation of China (Grant No. U1664264), the China Fundamental Research Funds for the Central Universities (Grant No. 300102228402, 3100102229103 and
300102229111), Xi'an Social Science Planning Fund (Grant No. 19Z73) and the Postgraduate Scientific Research Practice Innovation Project of Chang'an University (Grant No. 300103002046).

This is an Open Access article distributed under the terms of the Creative Commons Attribution License

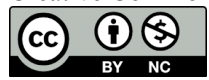

\section{References}

1. Zhen, F. G., Yang, L., Xiao, D. J., "The electric vehicle routing problem with time windows using genetic algorithm". In: Advanced Information Technology, Electronic \& Automation Control Conference, Chong Qing, China: IEEE, 2017, pp.691-695.

2. Maurizio, B., Ferdinando, P., Ornella, P., "A variable neighborhood search branching for the electric vehicle routing problem with time windows". Electronic Notes in Discrete Mathematics, 47, 2015, pp.221-228.

3. Dantzig, G. B., Ramser, J. H., "The truck dispatching problem". Management Science, 6(1), 1959, pp.80-91.

4. Golden, B., Assad, A., Levy, L., "The fleet size and mix vehicle routing problem". Computers \& Operations Research, 11(1), 1984, pp. 49-66.

5. Grosso, R., Muñuzuri, J., Escudero-Santana, A., "Mathematical formulation and comparison of solution approaches for the vehicle routing problem with access time windows". Complexity, 2018(7), 2018, pp.1-10.

6. Naccache, S., Côté, Jean-François., Coelho, L. C., "The multipickup and delivery problem with time windows". European Journal of Operational Research, 269(1), 2018, pp. 353-362.

7. Xie, J. Y., Fu, Z., Qiu, M., "A tabu search algorithm for vehicle routing problem with multiple soft time windows". Computer Engineering and Applications, 55(06), 2019, pp.78-86.

8. Wu, Z. Y., Lu, G. Y., Ma, S., "An optimization model for vehicle routing with multi-resource constraints". Journal of Transportation Engineering and Information, 16(01), 2018, pp.122-130.

9. Xu, H., Pu, P., Duan, F., "Dynamic Vehicle Routing Problems with Enhanced Ant Colony Optimization". Discrete Dynamics in Nature \& Society, 2018, 2018, pp.1-13.

10. Han, Y., Xue, N., Wang, B. Y., "Improved dual-protected ring signature for security and privacy of vehicular communications in vehicular Ad-Hoc networks". IEEE Access, 6, 2018, pp.1-1.

11. Zhang, J. H., Zhao, Y. X., "Vehicle routing problem with fuel consumption and carbon emission". International Journal of Production Economics, 170, 2015, pp.234-242.
12. Liu, C., Deng, K., Li, C., "The optimal distribution of electricvehicle chargers across a city". In: International Conference on Data Mining. Barcelona, Spain: IEEE, 2017, pp.261-270.

13. Keskin, M., Catay, B., "Partial recharge strategies for the electric vehicle routing problem with time windows". Transportation Research Part C: Emerging Technologies, 65, 2016, pp.111-127.

14. Yao, W. F., Wen, F. S., Zhao, J. H., Dong, Z. Y., "A multiobjective collaborative planning strategy for integrated power distribution and electric vehicle charging systems". IEEE Transactions on Power Systems, 29(4), 2014, pp.1811-1821.

15. Erdoğan, S., Miller-Hooks, E., "A green vehicle routing problem". Transportation Research Part E Logistics \& Transportation Review, 48(1), 2012, pp.100-114.

16. Velázquez-Martínez, J. C., Fransoo, J. C., Blanco E. E., et al, “A new statistical method of assigning vehicles to delivery areas for CO2 emissions reduction". Transportation Research, Part D: Transport and Environment, 43D(Mar), 2016, pp.133-144.

17. Ahn, K., Rakha, H., "The effects of route choice decisions on vehicle energy consumption and emissions". Transportation Research Part D: Transport \& Environment, 13(3), 2008, pp.151167.

18. Miguel, F., "Vehicle routing problem for emissions minimization". Transportation Research Record Journal of the Transportation Research Board, 2197(2197), 2010, pp.1-7.

19. Asvin, Goel., "The value of in-transit visibility for supply chains with multiple modes of transport". International Journal of Logistics Research \& Applications, 13(6), 2010, pp.475-492.

20. Thangiah, S. R., Nygard, K. E., Juell, P. L., "GIDEO: A genetic algorithm system for vehicle routing with time windows". In: IEEE Conference on Artificial Intelligence Applications, Miami, USA: IEEE, 1, 1991, pp. 322-328.

21. Bullnheimer, B., Hartl, R. F., Strauss, C., "An improved ant system algorithm for the vehicle routing problem". Annals of Operations Research, 89, 1999, pp.319-328.

22. Lang, M. X., Hu, S. J., "Study on the optimization of physical distribution routing problem by using Hybrid Genetic Algorithm". Chinese Journal of Management Science, 15(3), 2002, pp.76-79. 